\title{
Invasive Fungal Infection by Hormographyella aspergillata: A Tricky Diagnosis Triggered by $(1,3)$-Beta-D-Glucan Assay
}

Raffaella Koncan $^{1 *}$, Gianpaolo Nadali², Vincenza Favuzzi ${ }^{3}$, Marco Ligozzi ${ }^{1}$, Annarita Sorrentino ${ }^{4}$ and Giuliana Lo Cascio ${ }^{4}$

${ }^{1}$ Microbiology and Virology Unit, Department of Diagnostic and Public Health, University of Verona, Verona, Italy

${ }^{2}$ Hematology Section, Department of Clinical and Experimental Medicine, University of Verona, Italy

${ }^{3}$ Pathological Anatomy Unit, Department of Diagnostic and Public Health, University of Verona, Verona, Italy

${ }^{4}$ Microbiology and Virology Unit, Department of Pathology, Integrated University Hospitals of Verona, Italy

\begin{abstract}
We report on a pulmonary infection caused by the basidiomycete Hormographiella aspergillata. For the first time, $(1,3)$-beta-D-Glucan serum levels were monitored and they progressively increased during the development of clinical symptoms of infection. This assay and subsequent molecular analysis resulted essential for accurate identification and indication of proper antifungal therapy.
\end{abstract}

Keywords: (1,3)-beta-D-glucan; Rare fungal pathogen; Pulmonary fungal infection; Hormographyella aspergillata; Molecular diagnostics

\section{Abbreviations}

BM: Basidiomycetes; BG: (1,3)-beta-D-glucan; CBP: Clinical Breakpoints; CT: Computed Tomography; GM: Galactomannan; IFI: Invasive Fungal Infection; MIC: Minimum Inhibitory Concentration; MPAL: Mixed Phenotype Acute Leukemia; PCR: Polymerase Chain Reaction

Hormographiella aspergillata is a Basidiomycete; it belongs to the Psathyrellaceae family. It is the asexual form of Coprinopsis cinerea which is normally found in compost and sewage. The potential of common environmental filamentous basidiomycetes (BM) as human pathogens in infections has not yet been examined under laboratory conditions; instead, BM from clinical specimens usually present non-sporulating mold that grow as cottony white colonies [1]. It has been rarely found in humans as pulmonary and skin infections, endocarditis, endophthalmitis, chronical sinusitis [2] but the incidence of human disease caused by environmental fungi has been on rise. This is primarily attributed to risk factors such as wide use of immunosuppressive drugs, invasive medical instrumentation, large-scale usage of azole antifungals in agriculture [1].

The most of reported cases of $H$. aspergillata infection occurred in patients with hematologic malignancies. The first case was documented in 1971 and the second twenty six years later. Subsequently, the number of cases grew up to a total of fourteen reported in the following fifteen years [1-3]. This increasing incidence might be due to the higher number of immunocompromised patients, but lack of proper identification is probably the main reason for the low number of observations. This is the first case described in Italy.

\section{Case Presentation}

On May 2014, 26 years old male of south Asian ethnic origin, was diagnosed with Mixed Phenotype Acute Leukemia (MPAL). MPAL is a rare disease, representing only $3-5 \%$ of acute leukemia of all age groups, and $2.4-3.7 \%$ in children [4]. It affects adults more frequently than children, with a slight male preference. The prognosis for MPAL is poor comparing to other acute leukemia, with an overall survival of eighteen months [5].

The patient was admitted in the Hematology Unit of the University Hospital of Verona and treated accordingly to an acute myeloid leukemia chemotherapy induction program with Fludarabine, Cytarabine and Idarubicin, (FLAI regimen).
On August 2014, the patient received a second FLAI chemotherapy course. Since Quantiferon test resulted positive, isoniazid prophylaxis was provided. Treatment was complicated by septic shock, sustained by Escherichia coli and multidrug resistant Morganella morganii, completely solved by carbapenems and aminoglicosides. During hospitalization, the patient received antifungal prophylaxis using posaconazole oral solution. However, during the second course of chemotherapy, posaconazole was interrupted because of nausea and vomiting.

Fifteen days after chemotherapy, the patient developed intense spontaneous pain in the left scapular area. Chest X-Rays were persistently negative up to day nineteen. At day twenty, thoracic computed tomography (CT) scan revealed two lesions compatible with invasive fungal infection (IFI), with a possible abscess involving the soft tissues of the thoracic wall (Figure 1).

The patient underwent thoracotomy with atypical resection of the left upper lobe and drainage of the abscess cavity [6]. The purulent material was analyzed. After $72 \mathrm{~h}$ on Sabouraud Dextrose agar (SDA) plates (Oxoid, Basingstoke, England), incubated at $30^{\circ} \mathrm{C}$, cottony white colonies grew. Identification was reached by microscopic examination of mycelium, stained with lactophenol-blue (Figure 2) but the precise diagnosis of Hormographiella aspergillata was confirmed by sequencing of ITS and D1-D2 region of ribosomal sub-unites $[7,8]$.

Spectrophotometric identification by MALDI-TOF (bioMerieux, SA, Marcy l'Etoile, France), did not lead to any result using SARAMIS software package (AnagnosTec).

Susceptibility assay was run by Sensititre YeastOne microplates (TREK Diagnostic System, Thermo Fisher Inc., West Sussex, UK)

*Corresponding author: Raffaella Koncan, Microbiology and Virology Unit Department of Diagnostic and Public Health, University of Verona, Verona, Italy, Tel: +39 045802 7190; Fax: + 39045802 7101; E-mail: raffaella.koncan@univr.it; raffaellak@yahoo.it

Received July 04, 2016; Accepted July 31, 2016; Published August 09, 2016

Citation: Koncan R, Nadali G, Favuzzi V, Ligozzi M, Sorrentino A, et al. (2016) Invasive Fungal Infection by Hormographyella aspergillata: A Tricky Diagnosis Triggered by (1,3)-Beta-D-Glucan Assay. J Microb Biochem Technol 8: 361-363. doi: 10.4172/1948-5948.1000309

Copyright: $\odot 2016$ Koncan R, et al. This is an open-access article distributed under the terms of the Creative Commons Attribution License, which permits unrestricted use, distribution, and reproduction in any medium, provided the original author and source are credited. 
and minimum inhibitory concentration (MIC) showed the following results: Fluconazole 16, voriconazole 0.03 , posaconazole 0.125 , amphotericin B 0.12 . No clinical breakpoints (CBP) are available for this genus of mold, but the strain was defined resistant to fluconazole, susceptible to voriconazole, posaconazole, amphotericin B, according to the last EUCAST and CLSI.

From the first septic episode to the last physical examination in our hospital, the patient was continually monitored for fungal antigenic galactomannan (GM) (Platelia ${ }^{\mathrm{TM}}$ Aspergillus Ag, Bio-Rad, MarnesLa-Coquette, France) and (1,3)-beta-D-glucan (BG) assays (Fungitell, Associates of Cape Cod Inc., E. Falmouth MA, USA). Results are summarize in Graph 1.

It is noteworthy that the first BG test was positive seven days before CT imaging, thus suggesting IFI, even forty days before microbiological identification of the causative agent.

Conventional chest X-Rays were repeatedly negative but the rapid raise of BG serum level directed further investigation in the hypothesis of IFI. GM test was always negative, reaching a value of 0.4 , close to the cut-off of 0.5 , when BG was $>523 \mathrm{pg} / \mathrm{mL}$.

Intravenous voriconazole was the therapeutic choice. The pharmacological treatment was successful: BG showed progressively decreasing values and the patient did not show any clinical symptoms of IFI.

A bone marrow aspirate showed persistence of residual disease $(8 \%)$.

The patient decided to receive further treatment at his home town. He died of leukemia progression few weeks later.

\section{Discussion and Conclusion}

Aspergillus spp. represent the most common pathogens causing pulmonary infection in immunocompromised patients and is

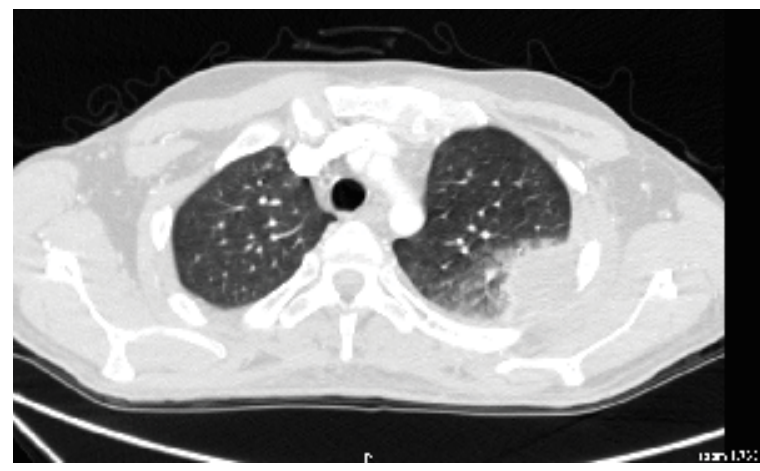

Figure 1: High-resolution CT scan of the lungs demonstrating a large nodule compatible with fungal infection.

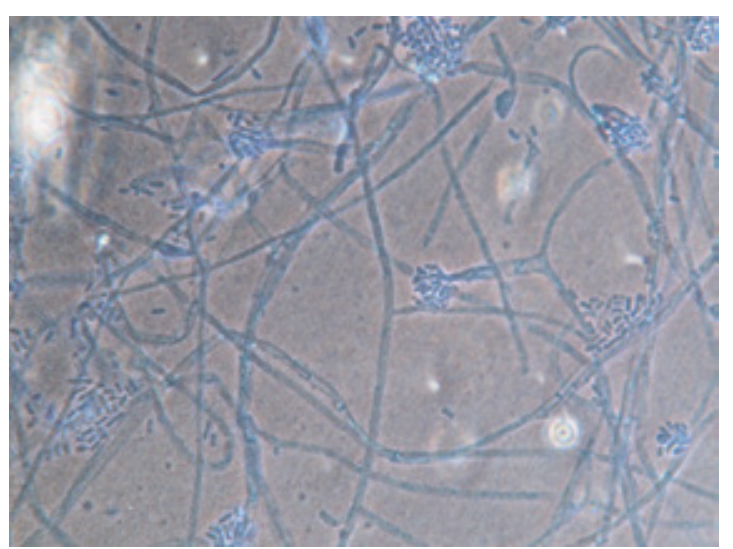

Figure 2: Lactophenol-blue stained preparation from culture: Septate, branched hyphe; conidiophores erect with basal septum often higher than supporting hypha; cylindrical arthroconidia with truncate ends but terminal cell rounded at the tip.

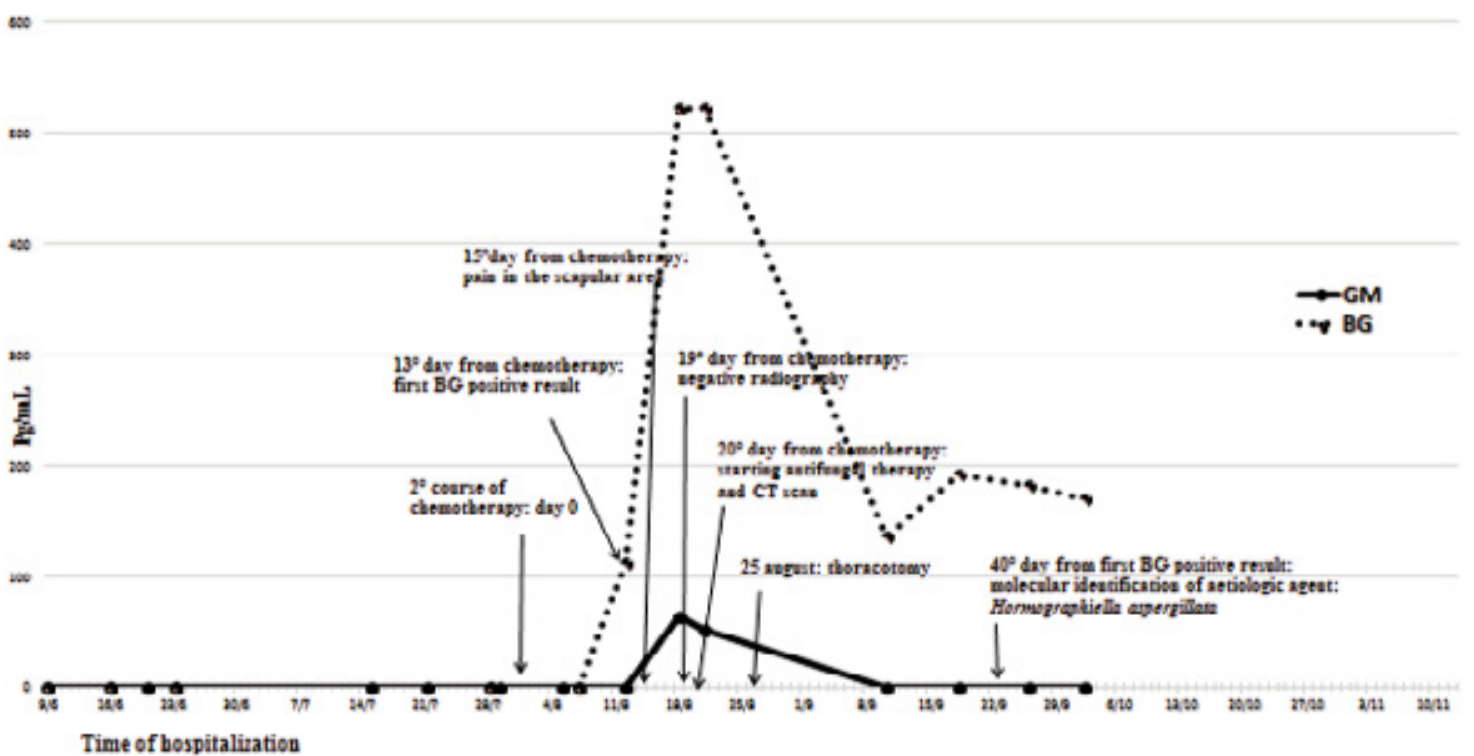

Graph 1: Results of different diagnostics assays performed during patient's hospitalization. (1,3)-beta-D-glucan (BG, dotted line) and galactomannan values (GM, solid line) were continually ran on patient's serum; on X axis days of hospitalization, on $Y$ axis BG and GM tests values. Values of BG and GM were proportionally adjusted on the base of cut off values, $80 \mathrm{pg} / \mathrm{mL}$ for BG, 0.5 for GM respectively. 
Citation: Koncan R, Nadali G, Favuzzi V, Ligozzi M, Sorrentino A, et al. (2016) Invasive Fungal Infection by Hormographyella aspergillata: A Tricky Diagnosis Triggered by (1,3)-Beta-D-Glucan Assay. J Microb Biochem Technol 8: 361-363. doi: 10.4172/1948-5948.1000309

usually revealed by the GM assay; in twelve cases of infection by Hormographiella GM was always negative, resulting of no diagnostic utility [1].

Identification of filamentous Basidiomycetes is difficult because key diagnostic features are often lacking, usually only arthroconidia are formed [9].

In Hormographiella aspergillata, also the shape of arthroconidia is non-diagnostics, therefore definitive identification requires sequencing of the ribosomal DNA [1]. The MALDI-TOF is still not useful: filamentous fungi are difficult to be treated by conventional protocols with formic acid and the database is still poor.

This is the first report on the use of a pan-fungal biomarker like $\mathrm{BG}$ to monitor the trend of infection by this rare pathogen: in our experience it has been useful to prompt diagnostic efforts in the identification of IFI.

It is known that Basidiomycetes are not susceptible to caspofungin, whereas Aspergillus spp. is susceptible to this antifungal drug: in one reported case, treatment for Aspergillus spp. was not effective for Hormographiella and the patient died [10]. Since very few documented cases have been observed, there is no established treatment for $H$. aspergillata; however the detection of the causative agent should be always pursued to avoid wrong therapy. In our opinion, BG should be considered a reliable diagnostic help in infections caused by Basidiomycetes.

\section{References}

1. Chowdhary A, Kathuria S, Agarwal K, Meis JF (2014) Recognizing filamentous basidiomycetes as agents of human disease: A review. Med Mycol 52: 782-797.
2. Bojic M, Willinger B, Rath T, Tobudic S, Thalhammer F, et al. (2013) Fata skin and pulmonary infection caused by Hormographiella aspergillata in a leukaemic patient: Case report and literature overview. Mycoses 56: 687-689.

3. Corzo-León DE, Satlin MJ, Soave R (2015) Epidemiology and outcomes of invasive fungal infections in allogeneic haematopoietic stem cell transplant recipients in the era of antifungal prophylaxis: A single-centre study with focus on emerging pathogens. Mycoses 58: 325-336.

4. Borowitz M, Bene MC, Harris NL, Porwit A, Matutes E (2008) Acute leukemias of ambiguous lineage. World Health Organization classification of tumours: Pathology and genetics of tumours of haematopoietic and lymphoid tissues, IARC Press, Lyon, France.

5. Matutes E, Pickl WF, Van't Veer M, Morilla R, Swansbury J, et al. (2011) Mixedphenotype acute leukemia: Clinical and laboratory features and outcome in 100 patients defined according to the WHO 2008 classification. Blood 117: 3163-3171.

6. Heiblig M (2015) Lyon HEMINF study group. Combined medico-surgical strategy for invasive sino-orbito-cerebral breakthrough fungal infection with Hormographiella aspergillata in an acute leukaemia patient. Mycoses 58: 308312.

7. White TJ, Bruns T, Lee S, Taylor JW (1990) Amplification and direct sequencing of fungal ribosomal RNA genes for phylogenetics. In: PCR Protocols: A Guide to Methods and Applications, Academic Press, New York.

8. Kurtzman CP, Robnett CP (1997) Identification of clinically important ascomycetous yeast based on nucleotide divergence in the 5' end of the large subunit (26S) ribosomal DNA gene. J Clin Microbiol 35: 1216-1223.

9. Lagrou K, Massonet C, Theunissen K, Meersseman W, Lontie M, et al. (2005) Fatal pulmonary infection in a leukaemic patient caused by Hormographiella aspergillata. J Med Microbiol 54: 685-688.

10. Conen A, Weisser M, Hohler D, Frei R, Stern M (2011) Hormographiella aspergillata: An emerging mould in acute leukaemia patients? Clin Microbiol Infect 17: 273-277. 